\title{
Influence of in-plane shear on kink-plane orientation in a unidirectional fibre composite
}

\author{
Wilhelmsson, D.; Mikkelsen, Lars Pilgaard; Fæster, Søren; Asp, L. E.
}

\section{Published in:}

Composites - Part A: Applied Science and Manufacturing

Link to article, DOI:

10.1016/j.compositesa.2019.01.018

Publication date:

2019

Document Version

Peer reviewed version

Link back to DTU Orbit

Citation (APA):

Wilhelmsson, D., Mikkelsen, L. P., Fæster, S., \& Asp, L. E. (2019). Influence of in-plane shear on kink-plane orientation in a unidirectional fibre composite. Composites - Part A: Applied Science and Manufacturing, 119, 283-290. https://doi.org/10.1016/j.compositesa.2019.01.018

\section{General rights}

Copyright and moral rights for the publications made accessible in the public portal are retained by the authors and/or other copyright owners and it is a condition of accessing publications that users recognise and abide by the legal requirements associated with these rights.

- Users may download and print one copy of any publication from the public portal for the purpose of private study or research.

- You may not further distribute the material or use it for any profit-making activity or commercial gain

- You may freely distribute the URL identifying the publication in the public portal 


\section{Accepted Manuscript}

Influence of in-plane shear on kink-plane orientation in a unidirectional fibre composite

D. Wilhelmsson, L.P. Mikkelsen, S. Fæster, L.E. Asp

PII:

$$
\text { S1359-835X(19)30017-X }
$$

DOI: https://doi.org/10.1016/j.compositesa.2019.01.018

Reference:

$$
\text { JCOMA } 5313
$$

To appear in:

$$
\text { Composites: Part A }
$$

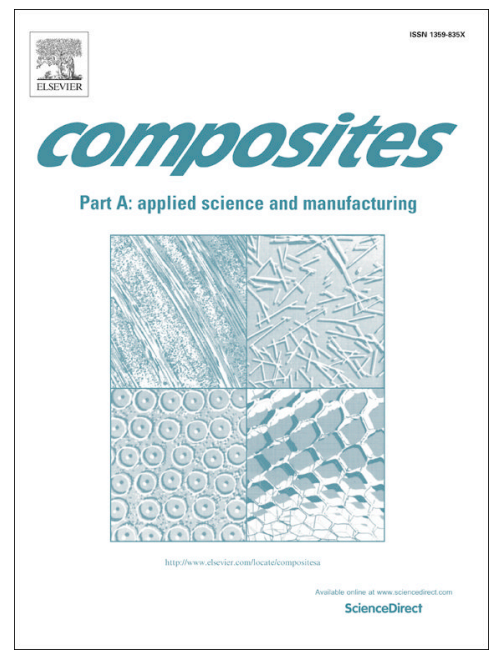

Received Date: $\quad 2$ October 2018

Accepted Date: $\quad 20$ January 2019

Please cite this article as: Wilhelmsson, D., Mikkelsen, L.P., Fæster, S., Asp, L.E., Influence of in-plane shear on kink-plane orientation in a unidirectional fibre composite, Composites: Part A (2019), doi: https://doi.org/10.1016/ j.compositesa.2019.01.018

This is a PDF file of an unedited manuscript that has been accepted for publication. As a service to our customers we are providing this early version of the manuscript. The manuscript will undergo copyediting, typesetting, and review of the resulting proof before it is published in its final form. Please note that during the production process errors may be discovered which could affect the content, and all legal disclaimers that apply to the journal pertain. 


\title{
Influence of in-plane shear on kink-plane orientation in a unidirectional fibre composite
}

\author{
D. Wilhelmsson ${ }^{\mathrm{a}}$, L. P. Mikkelsen ${ }^{\mathrm{b}}$, S. Fæster ${ }^{\mathrm{b}}$, L.E. Asp ${ }^{\mathrm{a}, *}$ \\ ${ }^{a}$ Industrial and Materials Science, Chalmers University of Technology, Hörsalsvägen $7 A$, \\ SE-41296, Göteborg, Sweden \\ ${ }^{b}$ Composite Mechanics and Structures, DTU Wind Energy, Technical University of Denmark, \\ Frederiksborgvej 399, DK-4000, Roskilde, Denmark.
}

\begin{abstract}
Kink-band formation is the governing failure mechanism for compressive failure of fibre reinforced composites. Here, kink-plane orientation, describing the direction of kink-band formation, is studied using X-ray computed tomography (CT). Unnotched unidirectional specimens with off-axis angles ranging from $0-20^{\circ}$ are tested in compression. The measured compressive strength is found practically constant for off-axis angles between $0-10^{\circ}$. For an off-axis angle of $15^{\circ}$ the compressive strength drops dramatically. CT-results reveal this drop to be consistent with a transition from out-of-plane to in-plane dominated kinking. Furthermore, results show the kink-plane angle to be linearly dependant on off-axis angle, and hence in-plane shear stress. A three-dimensional finite element model considering measured fibre misalignment angles through its volume is generated for numerical analysis. Numerical predictions based on the LaRC05 kinking criterion are found to qualitatively capture the experimentally observed effects of off-axis angle on kink-plane orientation.

Keywords: A. Carbon Fibres, C. Finite element analysis (FEA), D. CT analysis,

\footnotetext{
${ }^{*}$ Corresponding author

Email addresses: denwil@chalmers.se (D. Wilhelmsson), lapm@dtu.dk (L. P. Mikkelsen),
} sfni@dtu.dk (S. Fæster), leif.asp@chalmers.se (L.E. Asp)


D. Mechanical testing

\section{Introduction}

It is commonly known that the compressive strength of carbon fibre reinforced polymers is significantly lower than their tensile strength and much researeh is performed to address this problem. Basic research is needed to better understand the mechanics of compressive failure and for development of improved material models. The governing failure mechanism in compression loaded fibre reinforeed polymers is fibre kinking. Kink-bands develop when a cluster of fibres break from the loss of support of the surrounding polymer matrix. The support is lost due to matrix yielding and cracks caused by shear stresses. When a kink-band is formed fibres undergo rotations simultaneously in a local plane. This plane is referred to as the kink-plane and its orientation is studied in this paper.

As described in a recent review paper by Garcea et al. [1], the use of X-ray computed tomography $(\mathrm{CT})$ has grown significantly over the last decade. CT has enabled many types of study of composite materials that are simply not possible to perform by conventional microscopy. However, to the best of our knowledge only a few studies of compressive failure have used X-ray CT. Wang et al. [2] manufactured cylindrical rods with a diameter of $2.4 \mathrm{~mm}$ suitable for CT. The kink-bands were studied post-mortem. Geometric information of the kink-bands was obtained and information about the failure mechanisms within the kink-band was provided from tomographic reconstructions with high spatial resolution. CT monitored compressive tests have also been performed to study damage growth in-situ [3, 4]. The progression of a kink-band can occur with very high speed and an attempt to capture this using ultra-fast time-laps CT with a synchrotron X-ray source was made by Ueda et al. [3]. In a recent study by Emerson et al. [4], kink-band formation and growth in 
notched CFRP specimens was studied in-situ using ultra-fast time-laps CT. Tracking of individual fibres enabled characterisation of fibre orientations with a very high level of accuracy.

In this study we use X-ray CT as a tool to characterise the kink-plane with the ambition to provide detailed information on the transition from out-of-plane to inplane kinking in a unidirectional (UD) composite. The kink-plane angle describes in which direction the kink-band is progressing. For instance, at $90^{\circ}$ kinking occurs out of the plane and at $0^{\circ}$ kinking occurs in the plane of the laminate. Figure 1 depicts a CT image of a kink-plane in a specimen with an off-axis angle of $15^{\circ}$. The kink-plane in Fig. 1 is oriented at an angle between $0^{\circ}$ and $90^{\circ}$, i.e. somewhere between in-plane and out-of-plane.

Physically based failure criteria used in numerical models, such as LaRC05 [5], are dependent on an accurate prediction of the kink-plane angle. LaRC05's ability to predict the kink-plane angle for different off-axis angles is therefore evaluated by comparing finite element model predictions to CT measurements. Based on measured fibre misalignment data a novel material model is suggested.

Here, we study a unidirectional non-crimp fabric (NCF) carbon reinforced composite, a potential material for future aero-engine components. CT results from post-mortem analysis of unnotched, rectangular specimens from compression tests with different off-axis angles are presented. The variation of off-axis angles generates different levels of in-plane shear stress between laminates. By changing the magnitude of in-plane shear stress different kink-plane angles can be produced. 


\section{Method}

\subsection{Test specimens and mechanical compression tests}

The unidirectional test specimens consist of HTS45 [6] carbon fibres and LY556 [7] epoxy resin. The non-crimp fabric (NCF) woven preform is made from $12 \mathrm{k}$ carbon fibre warp yarns and polyamide/glass weft yarns as illustrated in Fig. 2. There are 2.4 bundles $/ \mathrm{cm}$ and 1 weft yarn/cm. The dry textile thickness is $0.3 \mathrm{~mm}$ and becomes approximately $0.2 \mathrm{~mm}$ in the processed UD composite with a fibre volume fraction of $53 \%$. The areal mass is $205 \mathrm{~g} / \mathrm{m}^{2}$ for the textile and $192 \mathrm{~g} / \mathrm{m}^{2}$ for the carbon fibres, calculated without sizing. Laminates were manufactured using the resin transfer moulding (RTM) process. Laminates with off-axis angles of $0^{\circ}, 5^{\circ}$, $10^{\circ}, 15^{\circ}$, and $20^{\circ}$ were made cutting the preforms before insertion into the RTM tool. A metal tool with dimensions $340 \mathrm{~mm} \times 240 \mathrm{~mm}$ (width $\times$ length) was used for manufacture of laminates with dimensions $327 \mathrm{~mm} \times 227 \mathrm{~mm}$ (width $\times$ length). The resin was infused along one edge at room temperature and a pressure of 2 Bar. The laminates were cured at maintained pressure at $80^{\circ} \mathrm{C}$ for $16 \mathrm{~h}$. Glass/epoxy laminate tabs with a thickness of $1.6 \mathrm{~mm}$ were adhesively bonded on each side before watercutting the specimens. The laminates presented in Table 1 (B2, D1-D4) were tested according to ASTM standards D6641 and D3410 [8, 9], with a nominal specimen size of $140 \mathrm{~mm} \times 12 \mathrm{~mm} \times 2 \mathrm{~mm}$ (length $\times$ width $\times$ thickness) and a gauge section length of $13 \mathrm{~mm}$. The effect of specimen width on compressive strength was analysed in a separate study [10]. It was concluded that a nominal width of $12 \mathrm{~mm}$ is sufficient to accurately measure the compressive strength of the off-axis laminates considered here. Furthermore, the maximum fibre misalignment angle out-of-plane, $\theta_{\max }$, which controls the compressive strength of the UD laminates (i.e. loaded along the fibres) was characterised by Wilhelmssson et al. [11]. The maximum out-of-plane misalign- 
ment angle was found to be $8.0^{\circ}$ for the studied UD laminate (i.e. of laminate B2 in Table 1). Measurements on one sample per off-axis angle in sections aligned with the local fibre orientations indicate very similar $\theta_{\max }$ values. Compressive tests were performed to characterise material strength. The CLC fixture used for ASTM D6641 was made at Swerea SICOMP according to the standard and the ITRII fixture used for tests according to ASTM D3410 was manufactured by Wyoming Test Fixtures. Tests were conducted at the Swerea SICOMP laboratory in Mölndal, Sweden, in a $100 \mathrm{kN}$ MTS 20/M load rig. The compressive load was applied at a rate of $1.3 \mathrm{~mm} / \mathrm{min}$ until a drop in load was observed. Bending in the specimens was monitored with strain measurements on a few specimens from each laminate according to the standards $[8,9]$. Both standards allow for a maximum of $10 \%$ bending at failure for a test to be valid. It is noted in Table 2 that three out of five laminates exceeded this requirement. The issue of excessive bending has been thoroughly investigated in a previous study where it was concluded that it did not significantly affect the measured compressive strengths [11].

\subsection{X-ray micro-computed tomography}

One specimen for each off-axis angle was selected for post-mortem analysis with $\mathrm{X}$-ray CT. The scanned samples were in one piece, with the kink-band in the gauge section, as illustrated in Fig. 3. Two types of X-ray tomography scans were performed on a Zeiss Xradia 520 Versa on all the investigated laminates with the settings listed in Table 3. The first scan on each laminate was centered on the kink-band with a large field of view $($ FOV) of $13 \mathrm{~mm} \times 13 \mathrm{~mm}$ (FOV $13 \mathrm{~mm}$ ) to cover the full width of the sample providing an overview of the kink-band. The next scan with a small field of view of $3 \mathrm{~mm} \times 3 \mathrm{~mm}$ (FOV $3 \mathrm{~mm}$ ) was centered on the kink-band in the middle of the sample with respect to both width and thickness. This centering of the 
second scan was based on the reconstruction of the first scan using the scout-and-scan principle by Zeiss.

The X-ray energy used for both types of scans was polychromatic up to $30 \mathrm{kV}$ from a tungsten target and 5201 or 5801 projections were acquired during a full sample rotation of $360^{\circ}$ on a $2 \mathrm{k} \times 2 \mathrm{k}$ CCD. A binning of two was applied to obtain shorter scan time and better signal to noise ratio on the expense of lower resolution. Due to the different magnifications, the pixel size on the detector was $12.77 \mu \mathrm{m}$ and $3.02 \mu \mathrm{m}$ respectively in the two types of scan. The acquired $2 \mathrm{D}$ projections on the detector were reconstructed into 3D density maps by a Feldkamp algorithm for cone beam reconstruction $[12]$ to $1 \mathrm{k} \times 1 \mathrm{k} \times 1 \mathrm{k}$ pixel volumes.

\subsection{Experimental characterisation of $\psi$}

A kink-plane is schematically illustrated in Fig. 4 as a local plane on which deformation lead to the formation of a kink-band. The kink-plane angle $\psi$ is defined relative to the $\mathrm{X}-\mathrm{Y}$ plane and can obtain a value between $0^{\circ}$ and $180^{\circ}$. An angle $\psi$ of $90^{\circ}$ represents a kink-band oriented out of the plane and an angle $\psi$ of $0^{\circ}$ (or $180^{\circ}$ ) means that the kink-band is oriented in the plane. We refer to these two extreme cases as "out-of-plane kinking" and "in-plane kinking" respectively.

Characterisation of the kink-plane angle $\psi$ in compression loaded UD-laminate specimens with off-axis angles $0^{\circ}, 5^{\circ}, 10^{\circ}, 15^{\circ}$, and $20^{\circ}$ is performed on scans with FOV $13 \mathrm{~mm}$ covering the whole domain of the kink-bands. The kink-plane angle $\psi$ is measured on sections in the YZ plane as illustrated in Fig. 5. The regions containing damage in Fig. 5 represents a cut through the kink-band, which extends in a direction normal to the kink-plane. A sample with $15^{\circ}$ off-axis angle is shown in Fig. 6. The local coordinate system 1-2 is along the fibre direction with a $15^{\circ}$ off-axis angle to the laminate coordinate system X-Y. As shown in Fig. 6, the angle $\psi$ is measured at 
10 locations over the kink-band with equal spacing in the longitudinal direction and constant position in the thickness of the specimens. With multiple measurement points, the variation of $\psi$ can be determined. This method to characterise $\psi$ is referred to as the "CT method".

The kink-plane angle is also characterised by an alternative method. Two specimens from each off-axis angle are characterised with an optical microscope in terms of the kink offsets according to Fig. 4. These offsets are measured from micrographs obtained with the specimens oriented in-plane and out-of-plane as viewed in Fig. 3. The average in-plane and out-of-plane values for each off-axis angle are used to calculate $\psi$ as $\tan$ (kink offset out-of-plane / kink offset in-plane). The angle $\psi$ measured by the kink-offset method represents the average angle for a kink-band since the macroscopic displacements are characterised for the laminate as illustrated in Fig. 4. This method to characterise $\psi$ is referred to as the "kink-offset method".

\subsection{Numerical modelling}

The finite element method (FEM) is used to predict the kink-plane angle $\psi$ for the different off-axis angles. Based on measured fibre misalignment data a novel approach is presented. The spatial resolution of measured fibre misalignments is $100 \mu \mathrm{m}$. Experimentally characterised fibre waviness is mapped on a finite element mesh in Abaqus as local material orientations, similar to the work by Wilhelmsson et al. [13]. In this approach we use one in-plane and one out-of-plane 2D section respectively (Fig. 7) and map these on a 3D mesh representing the gauge section (Fig. 8). We assume that the in-plane fibre misalignments are constant through the thickness and that the out-of-plane fibre misalignments are constant over the width. The fibre orientation for each element becomes a combination of the in-plane, outof-plane and off-axis angle. Thus, a unique misalignment angle is given for each 
element. A total of 237,620 different fibre angles are present in the model for each off-axis case representing the $3 \mathrm{D}$ waviness. Each off-axis case $0^{\circ}, 5^{\circ}, 10^{\circ}, 15^{\circ}$ and $20^{\circ}$ contain the same misalignments as in Fig. 7 but with different misalignments from the off-axis angles added to these.

We have conducted this study with the hypothesis that fibre kinking can be studied and modelled from 2D sections. More specifically on 2D sections aligned with the kink-band. With the assumption of constant out-of-plane misalignment angles over the width, we have considered one sample of out-of-plane misalignment angles. Also, with the assumption of constant in-plane misalignment angles over the thickness, we have considered one sample of in-plane misalignment angles. It is thus a quasi 3D modelling approach that we have chosen in this study. A larger combination of samples is needed for more quantitative results.

The fibre misalignments of the 2D sections are characterised on micrographs with the HRMA method [14]. The HRMA method is based on tracing individual fibre segments directly on detailed micrographs. Fibre misalignment data of individual segments are averaged in cells, which determine the spatial resolution of the HRMA method. In this study, with a spatial resolution of $100 \mu \mathrm{m}, 6$ fibres per cell are measured in average. This give a total of approximately 70,000 measurements in-plane and 13,000 measurements out-of-plane. The weft yarns shown in Fig. 2 cause regions without misalignment data. For simplicity, the misalignment field is interpolated to fill these gaps.

The generated 3D domain is discretised with second order hexahedral elements with full integration (C3D20) and the size is $100 \mu \mathrm{m}$ to match the misalignment data discretisation. Mesh convergence has not been studied. However, the chosen meshsize is the same for all cases. Given that the FE results are of a qualitative nature generated to study the variation of the kink-plane orientation over the width and for 
different off-axis angles we consider the predicted trends reliable. The misalignment data is mapped directly by ${ }^{*}$ orientation and ${ }^{*}$ orientation table in conjunction with * solid section. The elastic properties of the homogenised UD composite material used in the model are presented in Table 4. The longitudinal elastic modulus $E_{11}$ is calculated by the rule of mixtures (ROM) for a fibre volume fraction of $53 \%$, using fibre and matrix stiffnesses of $240 \mathrm{GPa}$ and $3.2 \mathrm{GPa}$ respectively [6, 7]. We use the theoretical value for $E_{11}$ since the FE model considers the local fibre orientations. Note that the experimental value of the longitudinal stiffness contain the stiffness reduction caused by fibre waviness $[11,15]$. The transverse and shear moduli reported in Table 4 were characterised for the same material system by Bru et al. [15].

An elastic perturbation is given as a prescribed displacement on one face in the $\mathrm{X}$-direction of the specimen with the opposite face fixed. This simulates a rigid attachment and is not meant to represent the experimental clamping with high fidelity. A static simulation is performed with small deformation theory using an iterative solver. The LaRC05 failure criteria [5] is implemented as subroutine, which calculates a user defined variable (uvarm) with the kink-plane angle as output. The analysis is fully linear, i.e. no consideration is taken to shear non-linearity etc. The LaRC05 kinking criterion is implemented with zero initial fibre misalignment angle (denoted $\phi$ by Pinho et al. [5]) since the local material orientation is given. Failure is evaluated in the span of $\psi=0-180^{\circ}$ in increments of $1^{\circ}$. The longitudinal shear strength $S_{L}$ is 56.7 MPa [15].

The kink-plane angle is sampled from five paths across the specimen width as illustrated in Fig. 8. These paths are equally spaced along the $\mathrm{X}$-axis and positioned in the centre of the thickness. Predicted kink-plane angle on these paths varies and data processing is therefore performed in Matlab with the following steps: (i) Values of $\psi$ larger than $180^{\circ}$ are non-physical and are thus removed. (ii) Values of $\psi$ between 
90-180 are subjected to a phase shift $180-\psi$ as the experimental results only consider the span $0-90^{\circ}$. (iii) For each path a fifth order regression curve is fit to the data. With this approach we filter out local fluctuations and at the same time are able to describe the variation of $\psi$ over the width.

\section{Results and discussion}

\subsection{Experimental compression tests}

Laminates D1-D4 have the fibres oriented in off-axis angles in the interval 5-20 . When tested in compression all these laminates failed by kinking of the fibres. This confirms previous observations by Edgren et al. [16] and Shipsa et al. [17] who found multiaxial NCF composites to fail by kinking for off-axis angles up to $20^{\circ}$. Visual inspection and optical microscopy showed kinking to occur out-of-plane in all offaxis specimens. However, unlike the $0^{\circ}$ specimens, where kinking only took place out of the plane in the previous study [11], the off-axis specimens were found to have a component of kinking also in the plane. Furthermore, as the off-axis angle was increased, the amount of in-plane kinking increased as well.

The measured compressive strength, stiffness and strain to failure are presented in Table 5 with strength variations more clearly shown in Fig. 9. The compressive strength for laminate $\mathrm{B} 2\left(0^{\circ}\right)$ of approximately $400 \mathrm{MPa}$ is relatively low as a consequence of the high fibre waviness [11]. The compressive strength remains constant for off-axis angles of $5^{\circ}$ and $10^{\circ}$ and is found to drop for $15^{\circ}$. The compressive strength is further reduced to $258 \mathrm{MPa}$ for an off-axis angle of $20^{\circ}$. The coefficient of variation for strength $C V_{X_{C}}$ is between $9-13 \%$ for the $0^{\circ}, 5^{\circ}$ and $10^{\circ}$ laminates and reduced as the off-axis angle is further increased. A sudden drop in compressive strength from an increased off-axis angle was also observed in a recent study by Shipsa et al.[17] for an NCF composite. In that study it was noted to occur between $5^{\circ}$ and $10^{\circ}$. 
As expected the elastic compressive stiffness is reduced as the off-axis angle is increased. The elastic modulus of the $0^{\circ}$ laminate of $102 \mathrm{GPa}$ is reduced to $33 \mathrm{GPa}$ for an off-axis angle of $20^{\circ}$. Furthermore, the coefficient of variation for stiffness $C V_{E}$ is reduced as the off-axis angle is increased. This can be explained by a lower contribution from fibre waviness to the scatter for larger off-axis angles. Finally, the strain to failure was $0.39 \%$ for $0^{\circ}$ test samples and found to increase with an increase in off-axis angles up to $1.31 \%$ for $20^{\circ}$ off-axis angle. The stress-strain responses are not presented. They showed a typical linear relation up to failure for $0^{\circ}$ test specimens and became increasingly non-linear as the off-axis angle was increased.

\subsection{Experimental results for $\psi$}

Kink-plane angle $\psi$ as function of off-axis angle measured by the CT and kinkoffset methods are presented in Fig. 10. Since $\psi$ varies along the width, it is the mean value which is presented in Fig. 10 for CT and FEM results. The experimental results are plotted together with FEM predictions. Experimental results from the two methods are approximated with a first order least squares polynomial fit, also included in the graph. The relationship between $\psi$ and off-axis angle is found to be linear. As expected the kink-plane angle $\psi$ is decreasing with increased off-axis angle. That is, the kink-plane becomes more strongly oriented in the plane as the off-axis angle is increased. This is due to an increase in the shear stress component $\tau_{x y}$. The kink-plane is oriented out of the plane $\left(\psi \approx 90^{\circ}\right)$ for zero off-axis angle and is linearly reduced to $\psi \approx 25^{\circ}$ for an off-axis angle of $20^{\circ}$. In-plane kinking becomes geometrically dominant at an off-axis angle of approximately $13^{\circ}$ where $\psi$ falls below $45^{\circ}$. That is, the in-plane kink-offset (Fig. 4) becomes larger than the out-of-plane kink-offset (Fig. 4) at an off-axis angle of $13^{\circ}$.

With CT scans covering the full width of the specimens $(12 \mathrm{~mm})$, results presented 
here for the first time describe the spatial variation of the kink-plane angle $\psi$. In Fig. 11, the kink-plane angle $\psi$ is presented along the width. The results show $\psi$ to be relatively constant for lower off-axis angles with the kink-band mainly directed out-of-plane. However, for higher off-axis angles $\left(\geq 15^{\circ}\right)$ it varies significantly. The kink-plane angle $\psi$ varies in a similar manner for the samples with high off-axis angles. For these, the kink-band angle $\psi$ has its maximum in the centre of the specimens. The kink-plane has a stronger in-plane orientation at the specimen edges than in the centre. This is explained by in-plane shear as a longitudinal load-transferring mechanism at the free edges [10].

The kink-offset method does not provide any information on the variation of $\psi$. Nevertheless, as shown Fig. 10 it provides a simple and accurate methodology to measure the average kink-plane angle.

The kink-plane angle has been characterised post-mortem and not in-situ. However, recent results from notched circular specimens show that the kink-plane orientation is very similar from initiation to final failure [4], supporting the validity of the post-mortem analysis performed here.

Supplementary data is provided with this article in the form of 3D tiff stacks from the CT scans [18]. Supplementary data comprise both the $13 \mathrm{~mm}$ FOV readings covering the whole domain and $3 \mathrm{~mm}$ FOV scans covering the centre of the kinkbands. From the $3 \mathrm{~mm}$ FOV data, individual fibres are visible which enables detailed analysis of the kink-bands. Supplementary data is also provided as videos of the 3D tomographic reconstructions for each off-axis specimen [18].

\subsection{Numerical predictions of $\psi$}

Accurate modelling of compressive failure initiation and progression in composites depends on realistic predictions of the kink-plane angle $\psi$. In LaRC05 [5], the kink- 
plane angle is found by evaluating failure for different angles between $0-180^{\circ}$. The angle with the highest failure index determines the orientation of the kink-plane. In typical applications, the initial fibre misalignment $\phi$ has to be non-zero for the prediction of kinking, else the strength would be infinite. Here, however, we use a $\phi$ value of zero since the waviness is modelled with local material orientations.

In Fig. 10 the predicted kink-plane angle $\psi$ using FEM is plotted as function of off-axis angle together with the experimental results. The kink-plane angle $\psi$ is calculated as the mean along the five paths for each off-axis angle. The linear trend observed experimentally is well captured by the numerical predictions. The predicted reduction in kink-plane angle $\psi$ with increased off-axis angle is however smaller than found in experiments.

In Fig. 12 the predicted variation of $\psi$ over the width is presented. For higher off-axis angles, the FE model consistently predicts higher kink-plane angles in the middle than at the free edges. This agrees well with experimental observations. The trend of smaller variation of $\psi$ over the width for lower off-axis angles is also fairly well predicted.

Both the numerical model and the experimental measurements are based on one sample per off-axis angle. From a statistical point of view it is obviously difficult to fully evaluate the predictive capabilities of LaRC05. We can however conclude qualitatively that the main trends of how the kink-plane angle varies agree well with experimental results and thereby support the predictive capabilities of LaRC05.

\subsection{The effect of $\psi$ on compressive strength}

The reason for limited dependency of compressive strength on off-axis angles were studied and discussed in the 1990's [19]. The inconsistency between case (a) and case (b) in Fig. 13 in terms of compressive strength was the main concern. 
Why did a misalignment angle $\theta$ related to fibre waviness have a significant drop in strength and the same magnitude of misalignment $\theta$ associated with an uniform off-axis misalignment have a low effect? In this study with aid from state-of-the art characterisation methodology and numerical tools we are able to contribute with further knowledge on this topic.

Comparison between Figures 9 and 10 reveals that whereas the kinking angle varies linearly with increased off-axis angle the compressive strength is practically constant for the off-axis angles $0^{\circ}, 5^{\circ}$ and $10^{\circ}$ and suddenly drops as the off-axis angle reaches $15^{\circ}$. In an earlier study on the compressive strength of the UD composite material $\left(0^{\circ}\right)$ featuring a variation in out-of-plane fibre misalignment angles a strong correlation between the compressive strength and the maximum out-of-plane fibre misalignment angle was reported [11]. Furthermore, all these UD laminates loaded in the fibre direction failed by kinking out of plane. In contrast, the off-axis tests performed in the current study demonstrate a conspicuous competition between outof-plane and in-plane kinking. As shown in Fig. 10, as soon as an off-axis compressive load is applied the kink-plane orientation is changing to become more oriented in the plane. This successive alignment of the kink-plane with the XY-plane results from an increased shear stress acting in-plane. The experienced shear stress on the kink-plane as function of the off-axis angle thus increases as a consequence of both its increased alignment in-plane and the increased in-plane shear stress. In addition, shear stresses from a projection of the fibre waviness on the kink-plane are also acting on the kink-plane. A kink-band will initiate when the local shear strength in the kink-plane has been exceeded by these two sources of shear stress combined. Consequently, the contribution from the in-plane shear stress component caused by small off-axis angles will cause the kink-plane to rotate but may not affect the compressive strength significantly. The transition from out-of-plane to in-plane 
kinking with a sudden drop in strength can be viewed as a competition between shear stresses caused by out-of-plane fibre waviness and in-plane shear stresses caused by off-axis fibre misalignment. Consequently, higher fibre waviness out-of-plane will result in a delay of the transition.

As discussed above, fibre kinking occurs when the local shear strength on the kink-plane is exceeded. It depends mainly on the fibre misalignments and the stress state in the kink-plane. There are however a number of additional factors involved which affect the onset of fibre kinking. Firstly, we suggest that the difference in coupon thickness to width influence the kink-band formation. That is, more energy is needed to form a kink-band across the width than through the thickness of the test coupon. This will delay a shift from out-of-plane to in-plane kinking. Secondly, the studied composite is not transversely isotropic. The UD composite shows a significant difference in the out-of-plane shear and in-plane shear strengths. The out-of-plane shear strength $S_{13}$ is $56.7 \mathrm{MPa}$ whereas the in-plane shear strength $S_{12}$ is $77.8 \mathrm{MPa}$. Hence, to cause kinking in the plane, the higher in-plane shear strength value must be exceeded. This will further delay the shift from out-of-plane to inplane kinking. Only one longitudinal shear strength $\left(S_{L}\right)$ can be defined in the current version of the LaRC05 failure criteria and this is identified as a limitation considering the significant difference of $S_{13}$ and $S_{12}$ of the composite. Thirdly, it appears that waviness characteristics are different in-plane and out-of-plane, even though the maximum misalignment angles are similar. As reported by Wilhelmsson et al. [11] the waviness for laminate B2 is periodic out-of-plane whereas it is found here to be more randomly oriented in the plane. This observation is based on a micrograph of an in-plane section $(20 \mathrm{~mm} \times 12 \mathrm{~mm})$. Results show that a laminate with sinusoidal waviness has a lower compressive strength compared to laminates with the same maximum misalignment angle but with random waviness [11]. This 
will again postpone a shift from out-of-plane to in-plane kinking.

There is a correlation between the experimental error $C V_{X_{C}}$ and compressive strength $X_{c}$ presented in Table 5. By characterising the fibre waviness for multiple $0^{\circ}$ specimens, it was concluded that the variability in strength was mainly caused by the variability in fibre waviness [11], which is a defect in the material. For off-axis angles of $15-20^{\circ}$ failure is controlled by the magnitude of in-plane shear, which is related to the off-axis angle. Variability of the off-axis angle for different specimens within a laminate has not been quantified in this study but is expected to be significantly lower than the variability in fibre waviness. Consequently, the drop for $C V_{X_{C}}$ is related to a change in failure initiation from defect-controlled to in-plane shear-controlled. This is also supported by data in Table 5 where the local shear stress at failure $\tau_{12}^{u}$ for $15^{\circ}$ off-axis angle is $74 \mathrm{MPa}$, which is close to the in-plane shear strength of $77.8 \mathrm{MPa}[15]$.

\section{Conclusions}

The kink-plane angle $\psi$ describes the orientation of the plane in which the kinkband is forming. It is important to understand how it varies and how it relates to compressive strength. In this paper a dedicated experimental and numerical study of resulting kink-plane angles in an off-axis loaded UD NCF composite is presented. In particular, X-ray computed tomography results are presented offering detailed information on the local kink-plane orientation in the un-notched carbon fibre composite specimens.

The kink-plane angle is obtained by measurements of the inclination of the kinkband from slices in the YZ-plane. The kink-plane angle is oriented out of the plane $\left(\psi \approx 90^{\circ}\right)$ when the fibres are oriented in parallel to the external load (i.e. for an offaxis angle of zero degrees). The kink-plane then becomes more oriented in the plane 
as the in-plane shear is increased. The kink-plane angle is found to vary linearly with in-plane shear stress for off-axis angles between 0-20 . Furthermore, the kink-plane angle is found to be constant or vary significantly over the width depending on the off-axis angle. The kink-plane angle is dependent on the stress-state such that it is more aligned in-plane at the free-edges where in-plane shear stresses are higher.

A novel FEM approach to simulate the gauge section of a compression test specimen based on measured fibre misalignment data obtained with the HRMA method [14] is also presented. The FE model uses the LaRC05 failure criterion for kinking to evaluate the kink-plane angle in these specimens. The FE model is able to predict experimentally observed trends, such as the variation of $\psi$ with offaxis angle and the variation of $\psi$ over the width. The failure criterion considers only one shear strength $S_{L}$ along the fibres. This is identified to be a limitation of the LaRC05 criterion as the difference in out-of-plane and in-plane shear strength is significant for the studied material.

The compressive strength remains constant for off-axis angles of $5^{\circ}$ and $10^{\circ}$ and is found to drop significantly for $15^{\circ}$. This can be partly explained by the successive alignment of the kink-plane with the XY-plane, which results in an increased shear stress acting on the kink-plane. The drop in strength occurs when the shear stress from external loading, combined with the shear stress from fibre waviness, exceeds the local shear strength on the kink-plane. Additional factors which influence this transition are the laminate dimensions, difference in shear strength in-plane vs. outof-plane and the difference of fibre waviness in-plane vs. out-of-plane.

\section{Acknowledgements}

This work has been performed within the Swedish Aeronautical Research Program (NFFP), Project 2013-01119, jointly funded by the Swedish Armed Forces, 
Swedish Defence Materiel Administration, the Swedish Governmental Agency for Innovation Systems and GKN Aerospace. Sweden's innovation agency, VINNOVA, is also gratefully acknowledged for funding via LIGHTer Academy. The computations were performed on resources provided by Chalmers Centre for Computational Science and Engineering (C3SE). Peter Hällström performed major parts of the compressive testing at Swerea SICOMP and Love Roos conducted microsopy at Chalmers of the in-plane waviness. Their work is gratefully acknowledged.

This research was conducted using the Zeiss Xradia 520 Versa from the DTU Centre For Advanced Structural and Material Testing (CAS-MAT), grant no. VKR023193 from Villum Fonden.

\section{References}

[1] S. C. Garcea, Y. Wang, P. J. Withers, X-ray computed tomography of polymer composites, Composites Science and Technology 156 (2018) 305-319.

[2] Y. Wang, T. L. Burnett, Y. Chai, C. Soutis, P. J. Hogg, P. J. Withers, Xray computed tomography study of kink bands in unidirectional composites, Composite Structures 160 (2017) 917-924.

[3] M. Ueda, K. Mimura, T.-K. Jeong, In situ observation of kink-band formation in a unidirectional carbon fiber reinforced plastic by X-ray computed tomography imaging, Advanced Composite Materials 25 (1) (2016) 31-43.

[4] M. J. Emerson, Y. Wang, P. J. Withers, K. Conradsen, A. B. Dahl, V. A. Dahl, Quantifying fibre reorientation during axial compression of a composite through time-lapse x-ray imaging and individual fibre tracking, Composites Science and Technology 168 (2018) 47-54. 
[5] S. T. Pinho, R. Darvizeh, P. Robinson, C. Schuecker, P. Camanho, Material and structural response of polymer-matrix fibre-reinforced composites, Journal of Composite Materials 46 (19-20) (2012) 2313-2341.

[6] Teijin Toho Tenax, Technical data sheet, Delivery programme and characteristics for Tenax ${ }^{\circledR}$ HTS filamet yarn (2011).

[7] Huntsman, Technical data sheet, Araldite ${ }^{\circledR}$ LY556 / Aradur ${ }^{\circledR}$ 917/ Accelerator DY 070, Hot curing epoxy matrix system (2007).

[8] ASTM, ASTM D6641 - Standard Test Method for Compressive Properties of Polymer Matrix Composite Materials Using a Combined Loading Compression ( CLC ) Test Fixture, Tech. Rep. Clc, West Conshohocken, PA (2014).

[9] ASTM, ASTM D3410 - Standard Test Method for Compressive Properties of Polymer Matrix Composite Materials with Unsupported Gage Section by Shear, Tech. Rep. Reapproved 2008, West Conshohocken, PA (2008).

[10] D. Wilhelmsson, L. E. Asp, R. Gutkin, F. Edgren, Effect of specimen width on strength in off-axis compression tests, in: ECCM17, Munich, 2016.

[11] D. Wilhelmsson, R. Gutkin, F. Edgren, L. E. Asp, An experimental study of fibre waviness and its effects on compressive properties of unidirectional NCF composites, Composites Part A: Applied Science and Manufacturing 107 (2018) $665-674$.

[12] L. Feldkamp, L. Davis, J. Kress, Practical cone-beam algorithm, Journal of the Optical Society of America 1 (1984) 612-619. 
[13] D. Wilhelmsson, L. E. Asp, R. Gutkin, F. Edgren, Fibre waviness induced bending in compression tests of unidirectional NCF composites, in: ICCM21, Xi'an, China, 2017.

[14] D. Wilhelmsson, L. E. Asp, A high resolution method for characterisation of fibre misalignment angles in composites, Composites Science and Technology 165 (April) (2018) 214-221.

[15] T. Bru, P. Hellström, R. Gutkin, D. Ramantani, G. Peterson, Characterisation of the mechanical and fracture properties of a uni-weave carbon fibre/epoxy non-crimp fabric composite, Data in Brief 6 (2016) 680-695.

[16] F. Edgren, L. E. Asp, R. Joffe, Failure of NCF composites subjected to combined compression and shear loading, Composites Science and Technology 66 (15) (2006) 2865-2877.

[17] A. Shipsha, M. Burman, J. Ekh, Failure of cross-ply NCF composites under off-axis compressive loads - An experimental study and a new strength prediction model including fibre bundle waviness, Composites Part B: Engineering 153 (December 2017) (2018) 49-56.

[18] D. Wilhelmsson, L. P. Mikkelsen, S. F. Fæster, L. E. Asp, X-ray tomography data of compression tested unidirectional fibre composites with different off-axis angles (submitted), Data in Brief.

[19] M. R. Wisnom, Nonlinear analysis of misaligned unidirectional carbon fibreepoxy compression specimens, Composites Engineering 3 (6) (1993) 547-556.

[20] Porcher Composites, Technical data sheet, Style 4510 Finish F9773 (2014). 
Table 1: Basic properties of manufactured laminates where $n$ is the number of valid tests and $t$ is the thickness. The fibre volume fraction $V_{f}$ is calculated based on the areal weight and density of the fibres.

\begin{tabular}{lllll}
\hline Laminate & $n$ & Layup & $t(\mathrm{~mm})$ & $V_{f}(\%)$ \\
\hline B2 & 7 & {$[0]_{10}$} & 2.03 & 53 \\
D1 & 8 & {$[5]_{10}$} & 2.03 & 53 \\
D2 & 7 & {$[10]_{10}$} & 2.03 & 53 \\
D3 & 12 & {$[15]_{10}$} & 2.03 & 53 \\
D4 & 12 & {$[20]_{10}$} & 2.03 & 53 \\
\hline
\end{tabular}

Table 2: The number of specimens with strain gauges $n_{\epsilon}$, ITRII or CLC fixture and average bending at failure $\bar{B}_{f}$ within a laminate.

\begin{tabular}{llll}
\hline Laminate & $n_{\epsilon}$ & ITRII/CLC & $\bar{B}_{f}(\%)$ \\
\hline B2 & 7 & $1 / 6$ & 44 \\
D1 & 8 & $3 / 5$ & 24 \\
D2 & 7 & $2 / 5$ & 8 \\
D3 & 11 & $2 / 10$ & 16 \\
D4 & 11 & $2 / 10$ & 8 \\
\hline
\end{tabular}


Table 3: X-ray tomography settings.

\begin{tabular}{lll}
\hline Parameter & FOV $13 \mathrm{~mm}$ & FOV $3 \mathrm{~mm}$ \\
\hline Optical magnification & $0.4 \times$ & $4.0 \times$ \\
Source to sample dist. & $23 \mathrm{~mm}$ & $13 \mathrm{~mm}$ \\
Detector to sample dist. & $100 \mathrm{~mm}$ & $16 \mathrm{~mm}$ \\
Exposure time & $12 \mathrm{~s}$ & $10 \mathrm{~s}$ \\
No. of projections & 5201 & 5801 \\
Accelerating voltage & $30 \mathrm{kV}$ & $30 \mathrm{kV}$ \\
Binning & 2 & 2 \\
Pixel size & $12.77 \mu \mathrm{m}$ & $3.02 \mu \mathrm{m}$ \\
\hline
\end{tabular}

Table 4: Elastic material properties for the homogenised composite used in the FE model. Longitudinal stiffness $E$ and shear stiffness $G$ have the unit of GPa and Poisson's ratio $\nu$ is given in percent.

\begin{tabular}{ccccccccc}
$E_{11}$ & $E_{22}$ & $E_{33}$ & $\nu_{12}$ & $\nu_{13}$ & $\nu_{23}$ & $G_{12}$ & $G_{13}$ & $G_{23}$ \\
\hline 142 & 9.3 & 9.3 & 0.28 & 0.28 & 0.4 & 3.7 & 3.7 & 3.3 \\
\hline
\end{tabular}


Table 5: Summary of test data and associated scatter (CV) for compressive strength $X_{C}$, compressive elastic modulus $E$ and strain at failure $\epsilon_{c u}$. The local shear stress $\tau_{12}^{u}$ and normal stress $\sigma_{11}^{u}$ are calculated from a stress transformation based on the $X_{C}$ value and the off-axis angle.

\begin{tabular}{llllllll}
\hline Laminate & $X_{C}(M P a)$ & $C V_{X_{C}}(\%)$ & $E(G P a)$ & $C V_{E}(\%)$ & $\epsilon_{c u}(\%)$ & $\tau_{12}^{u}$ & $\sigma_{11}^{u}$ \\
\hline B2 $\left(0^{\circ}\right)$ & 394 & 13 & 102 & 16 & 0.39 & 0 & 394 \\
D1 $\left(5^{\circ}\right)$ & 405 & 9 & 88 & 10 & 0.48 & 35 & 402 \\
D2 $\left(10^{\circ}\right)$ & 394 & 12 & 65 & 5 & 0.72 & 67 & 382 \\
D3 $\left(15^{\circ}\right)$ & 297 & 7 & 43 & 8 & 0.95 & 74 & 277 \\
D4 $\left(20^{\circ}\right)$ & 258 & 3 & 33 & 5 & 1.31 & 83 & 228 \\
\hline
\end{tabular}

Figure 1: The kink-plane in a cross section through the tomographic reconstruction of the $15^{\circ}$ off-axis specimen. 


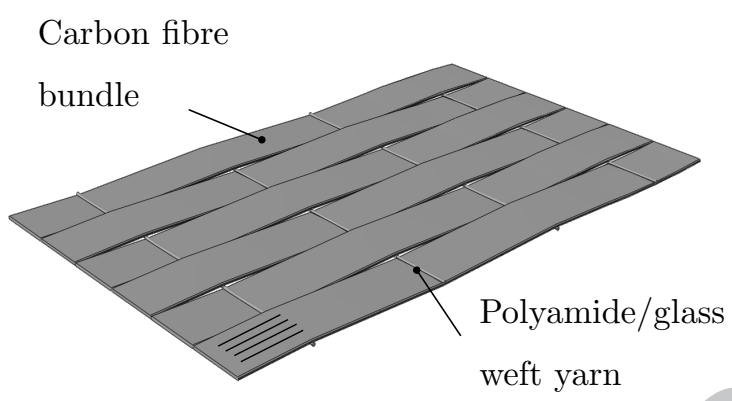

Figure 2: Illustration of the unidirectional NCF textile architecture produced by Porcher [20].

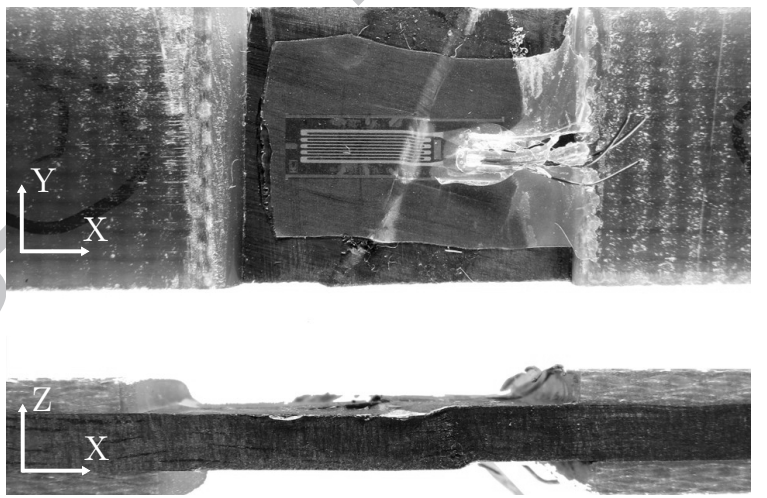

Figure 3: Gauge section of a $15^{\circ}$ specimen after testing. The kink-band stretches across both the width and the thickness of the specimen. 


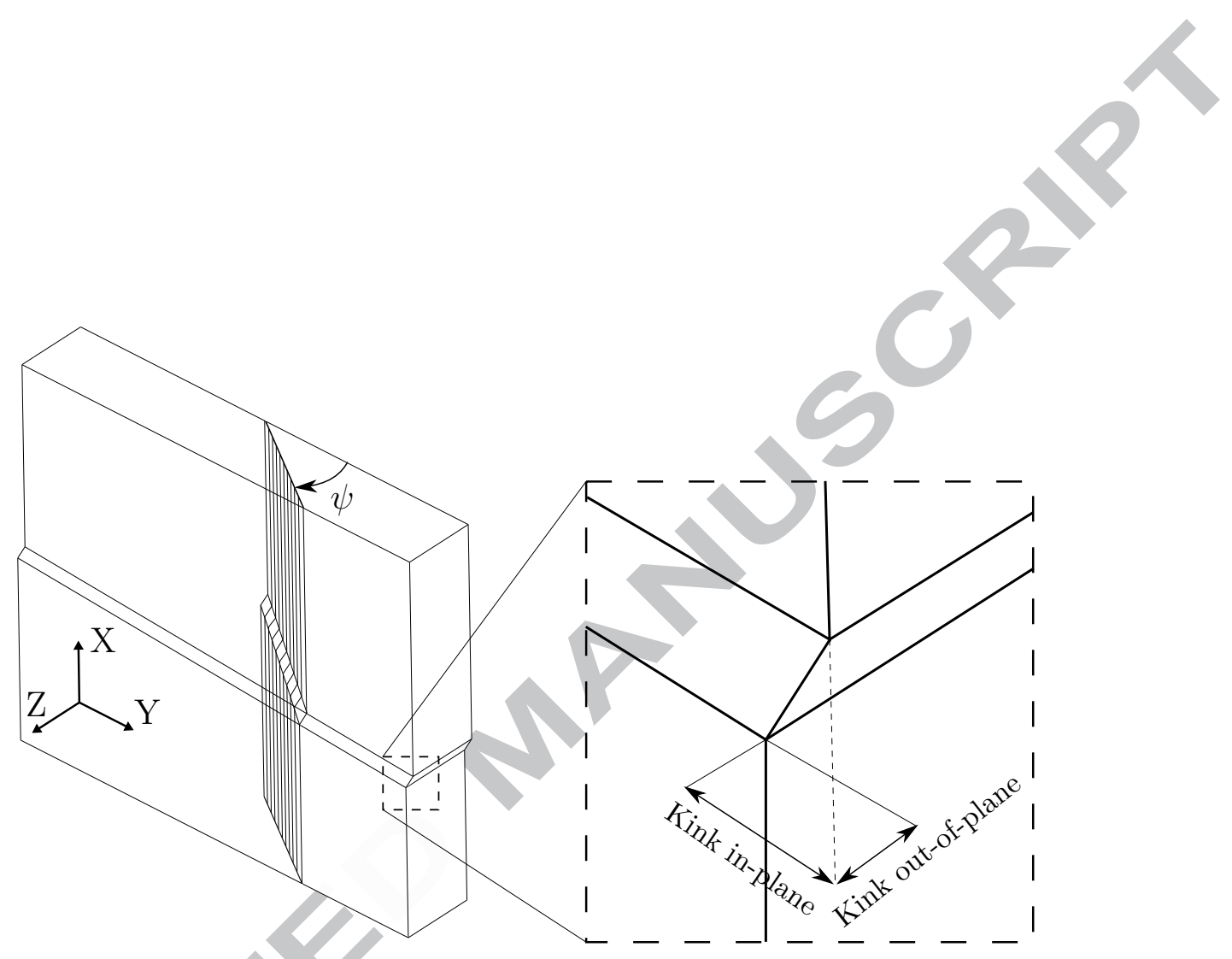

Figure 4: Schematic illustration of a kink-plane in 3D with the angle $\psi$. The enlarged region shows the components of kink (kink-offset) in the plane and out of the plane as a projection. 


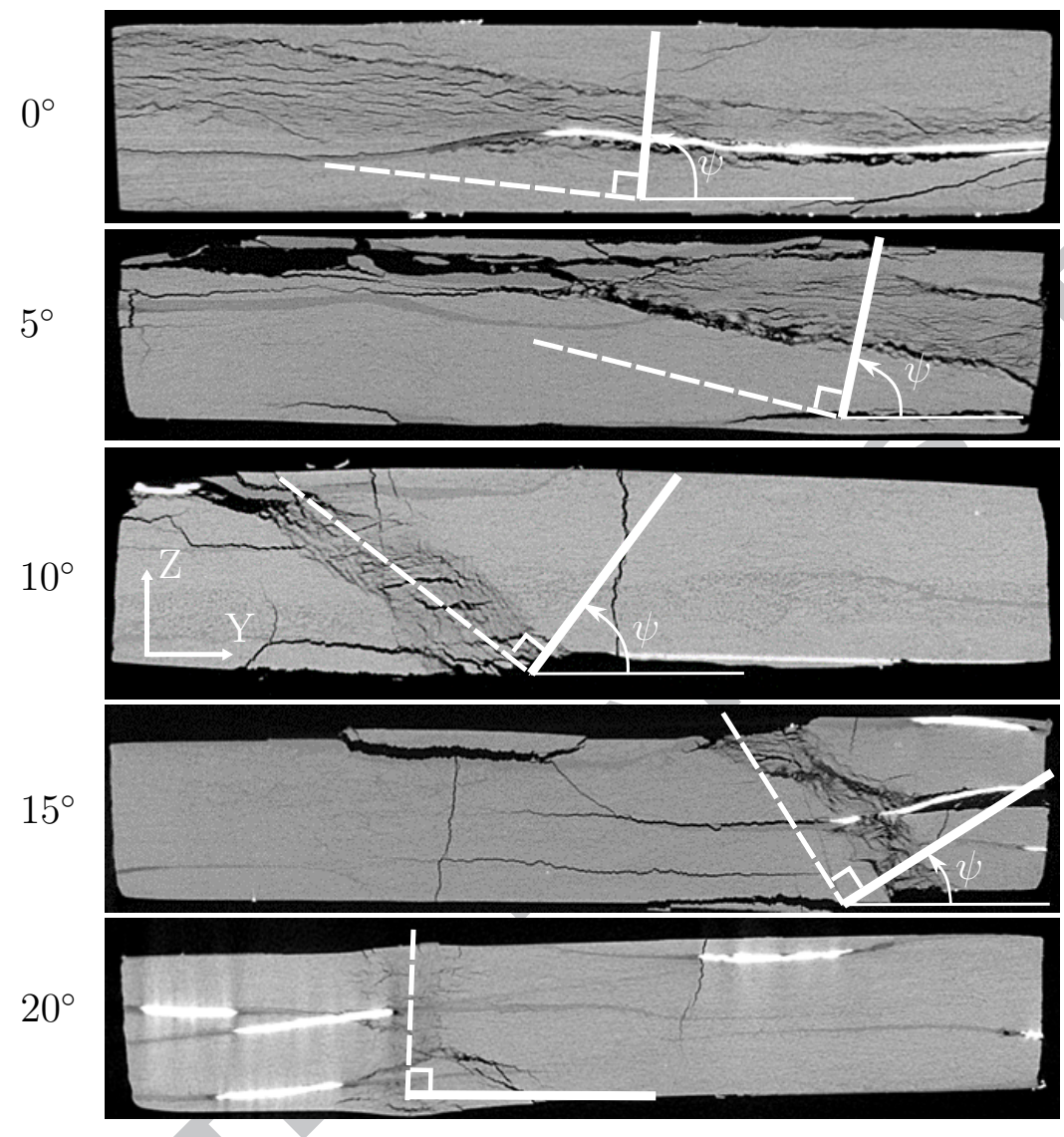

Figure 5: The kink-plane angle $\psi$ is obtained from CT images in the YZ plane. The specimens cross section are $12 \mathrm{~mm} \times 2 \mathrm{~mm}$. The solid bold line represents the kink-plane, the dashed line represents its normal and the solid thin line is an in-plane reference. Note that only one image is shown for each off-axis angle whereas 10 locations are used for the measurements. Videos of the 3D tomographic reconstructions are provided as supplemantary data [18]. Off-axis specimens $5^{\circ}$, $10^{\circ}$ and $15^{\circ}$ were tested with the CLC fixture [8] and the $0^{\circ}$ and $20^{\circ}$ specimens were tested with the ITRII fixture [9]. 


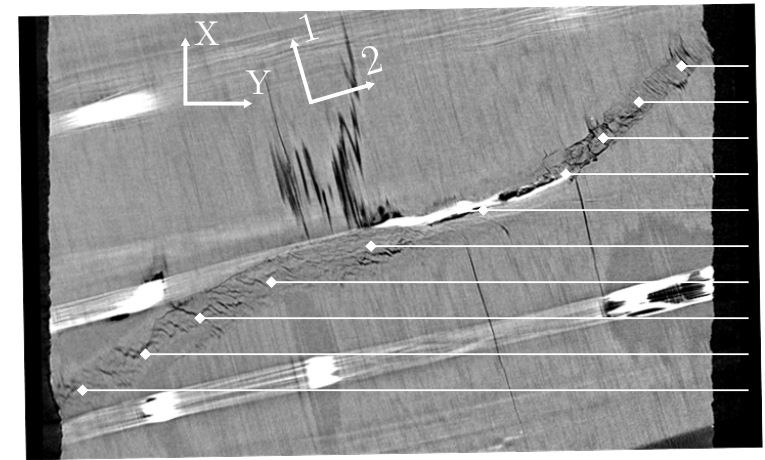

Figure 6: The kink-plane angle $\psi$ is characterised from CT images at 10 locations with equal spacing in the longitudinal direction (X-dir.) of the specimens. Here, a section is shown from the $15^{\circ}$ off-axis specimen.

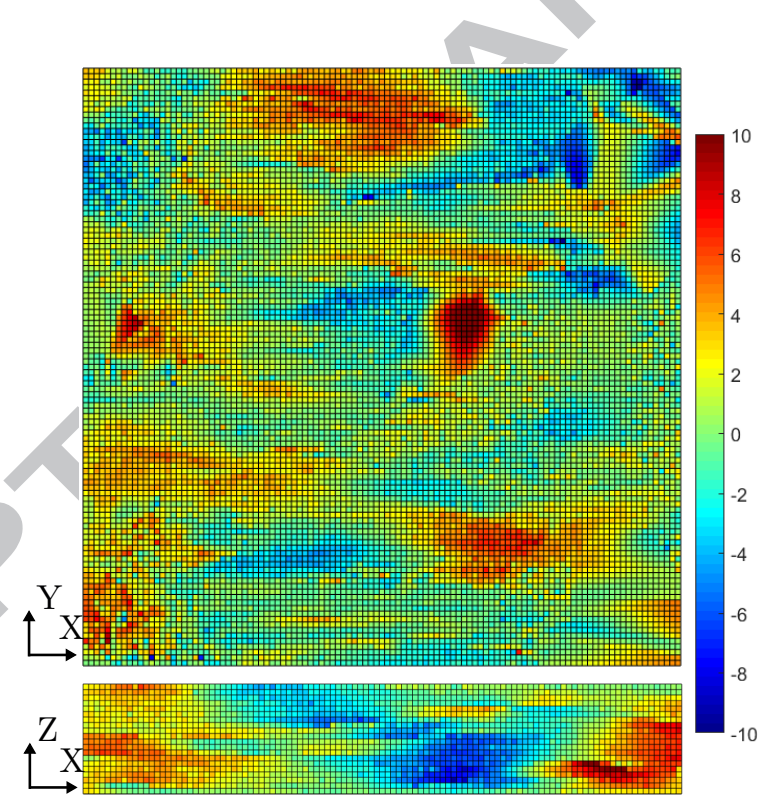

Figure 7: Experimentally characterised fibre misalignments with the HRMA method [14] inplane (upper) and out-of-plane (lower). The spatial resolution is $100 \mu \mathrm{m}$ and the domain size is $10.9 \mathrm{~mm} \times 10.9 \mathrm{~mm} \times 2 \mathrm{~mm}$ (longitudinal $\times$ width $\times$ thickness). 


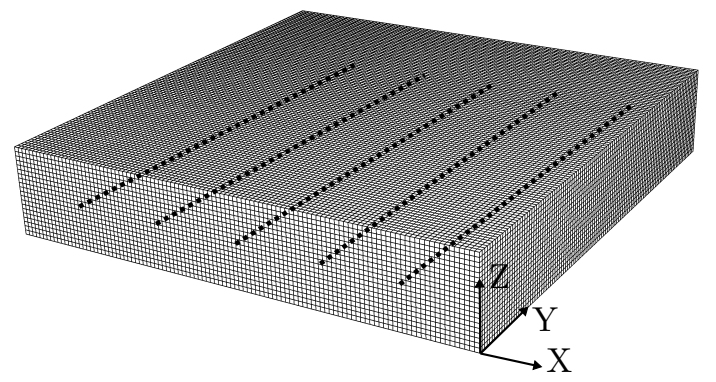

Figure 8: Finite element mesh with 5 paths for sampling of the kink-plane angle $\psi$.

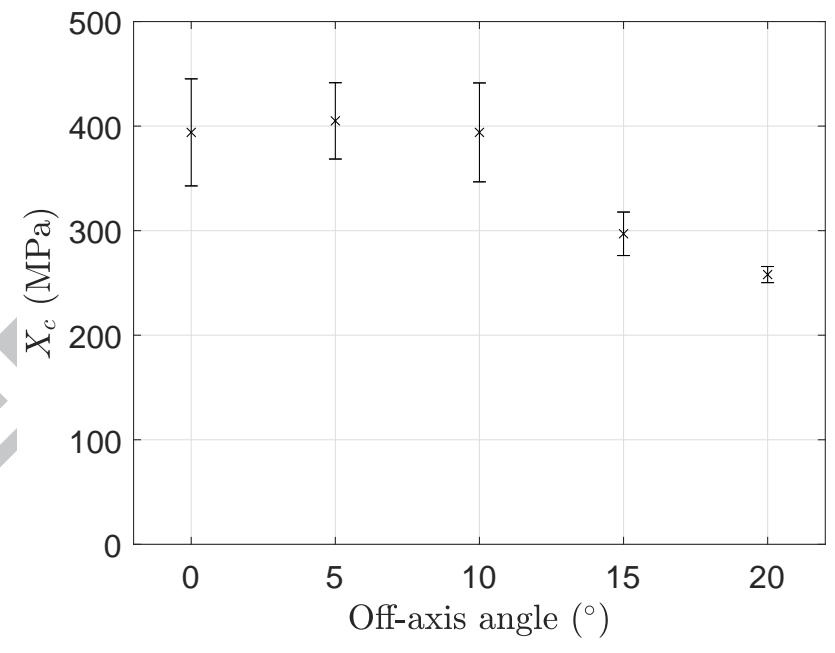

Figure 9: Compressive strength with associated standard deviations for different off-axis angles. 


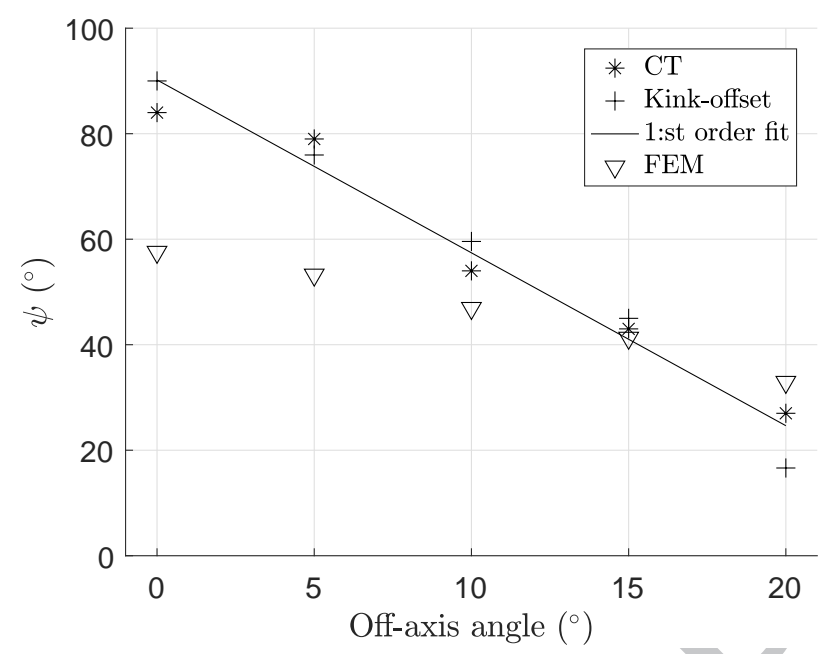

Figure 10: Kink-plane angle $\psi$ as a function of off-axis angle for measurements based on CT, kink-offset and FEM. The regression line is based on data from the CT and kink-offset methods

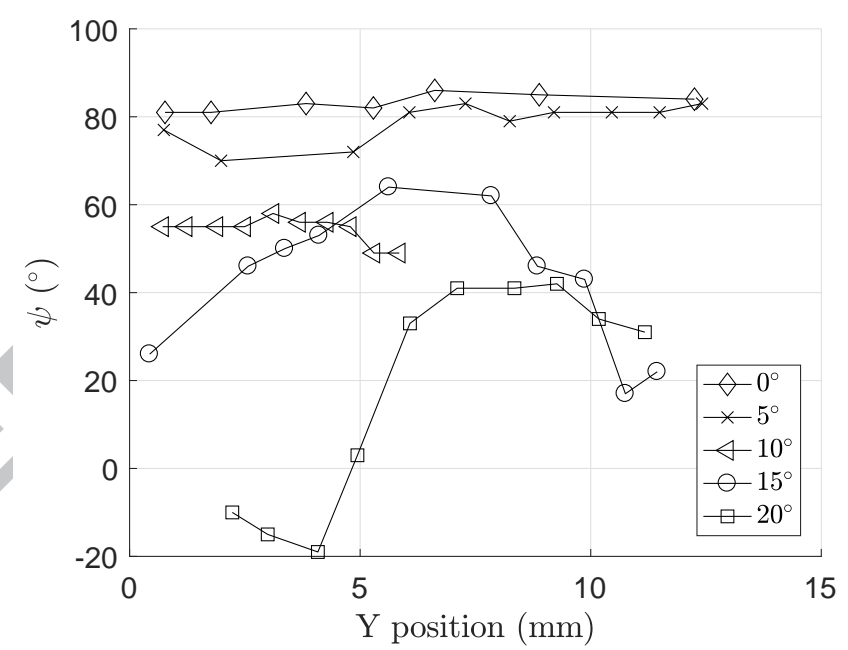

Figure 11: Variation of the kink-plane angle $\psi$ over the width of specimens with different off-axis angle based on CT results. Failure in the $10^{\circ}$ specimen is comprised of several shorter kink-bands where the most progressed is presented here. 

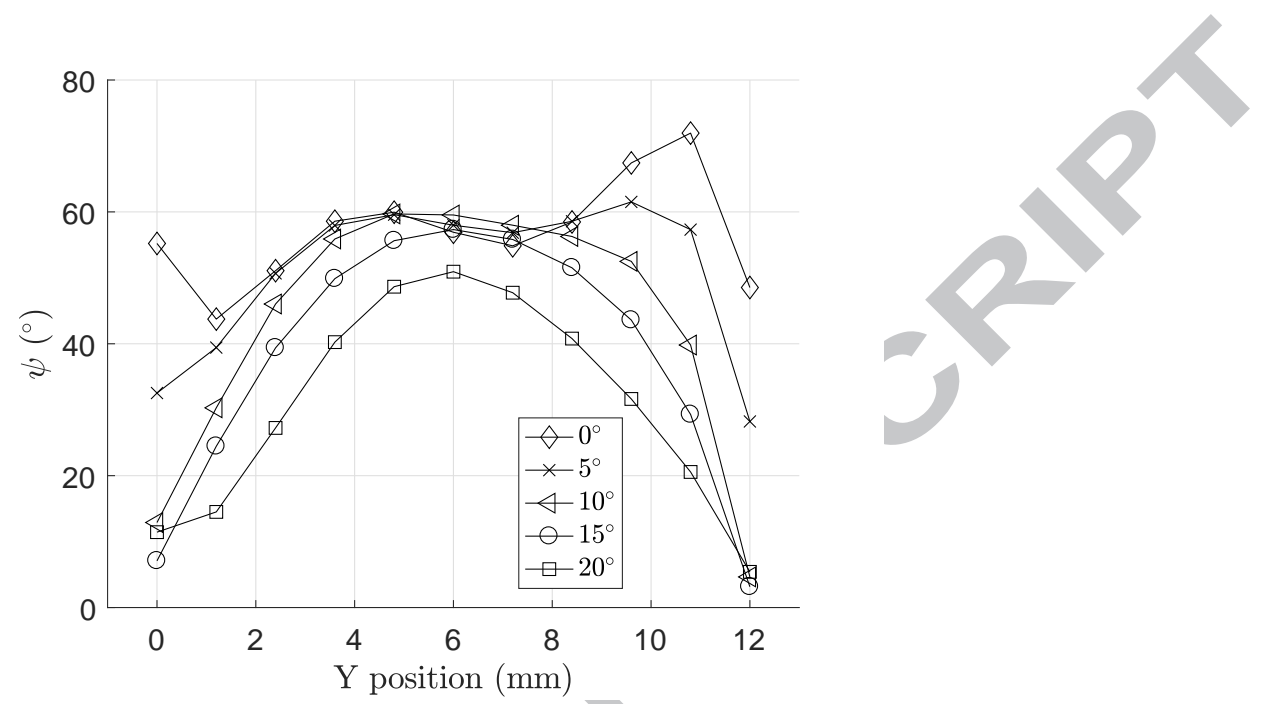

Figure 12: FEM predictions of the kink-plane angle $\psi$ over the width of specimens with different off-axis angles.

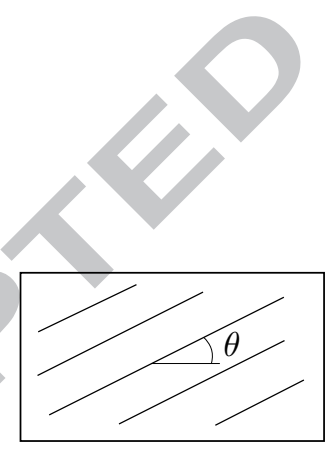

(a)

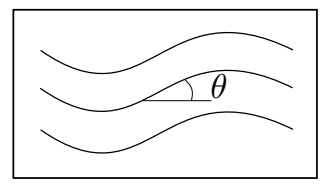

(b)

Figure 13: Fibre misalignment associated with (a) uniform misalignment (off-axis) and (b) fibre waviness. 